\section{Extreme Programming Refactored: The Case Against XP}

Matt Stephens and Doug Rosenberg

APress, 2003, \$39.99, ISBN: 1-590-59096-1

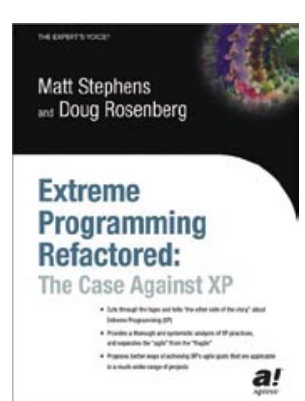

If you can tolerate the XSS (extreme satire and sarcasm) in this book, then you will find some very interesting and useful information about extreme programming - and also about the case against XP. There is a great deal of history, verbatim conversation, statements (usually e-mail), and descriptions about real projects.

Here and there, the authors point out that the book title implies that they have accepted XP, but are "tweaking and honing" it. The subtitle belies that claim. This paradox raises the deeper issues around when XP stops being XP. Does the "tweaking and honing" create something different-perhaps a more robust XP, as the authors claim-or an XP doomed to failure because its basic values, practices, and roles are compromised? Typical questions around this paradox are basic (and compelling) ones: Are there really exactly four values and 12 practices? Are they the right ones? How do we know?

The XSS is pervasive and is structured into the book. A story, "The Emperor's New Code," appears before the Preface, and "Satire Stories" are listed in a table of contents in the introduction, along with "Voice of Experience Stories" and "Songs of the Extremos." The experiences discussed are sometimes interesting, and the songs are entertaining adaptations of the Beatles (for example, "Hey Dude," "Test and Shout," "Eight Builds a Week"), Rolling Stones ("I Can't Get No Architecture"), and others.

There is a brief summary at the end of each chapter and an index at the end of the book. There is no bibliography, but there are embedded citations and footnotes, often of Web sites, user groups, lists, and e-mails.

The book has an unusual content and structure. The authors put a large amount of effort-and space-into the XSS and songs. Icons appear throughout the book alerting the reader to warnings, solutions, extremo quotes, satire and sarcasm, voices of experience, circular logic, and hype. All of this may not be attractive to the serious reader.-Susan M. Merritt

\section{Computer and Intrusion Forensics} George Mohay (Editor), Alison Anderson, Byron Collie, Olivier de Vel, and Rod McKemmish Artech House, 2003, \$79.00, ISBN: 1-580-53369-8

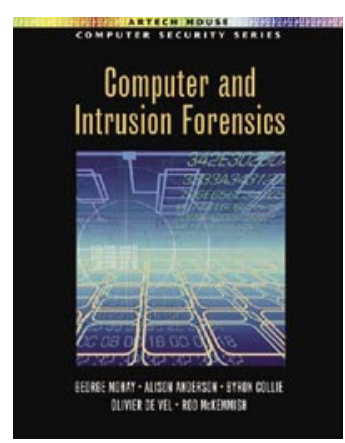

What could be more thrilling than solving a crime or determining if one occurred? This is certainly one of the reasons why computer forensics is a topic of interest to many, and one of the reasons why someone might purchase this book. However, readers should be warned that the book, written by five experts in the field with more than 80 years of forensic experience, sometimes has a dry and academic tone.

Part of the Artech House Computer Security Series, Computer and Intrusion Forensics will appeal to forensic investigators who are new to the field, including those in law enforcement, internal auditing, corporate security, and incident response. It is best suited for those who are seeking an overview of computer forensics, including managers and supervisors of forensic staff, the book offers broad coverage of the field.

The book covers requirements for establishing a case, digital evidence, types of forensic tools (disk, analysis, and visualization), and fundamentals of computer forensics. There also are overviews of fraud and forensic accounting and the two types of network detection systems, as well as a discussion of case studies that allow the reader to see how the tools and techniques discussed apply to the real world. Finally, the book covers a number of advanced topics: stegoforensic analysis, cryptography and cryptanalysis, data mining, and identifying e-mail authors.

This book contains valuable links to numerous Web sites. No CD-ROM, Web site, or additional material is provided by the publisher, so a reader who wishes to research further must be willing to search the provided URLs.

After completing Computer and Intrusion Forensics, readers should have a good understanding of the processes that occur during a computer investigation, but will still need additional training or study materials before they have the competency required to perform investigations themselves.-Joy Gumz Q

Reprinted from Computing Reviews, (C) 2004 ACM, http://www.reviews.com. 